\title{
Activated K-Ras and INK4a/Arf Deficiency Promote Aggressiveness of Pancreatic Cancer by Induction of EMT Consistent With Cancer Stem Cell Phenotype
}

\author{
ZHIWEI WANG ${ }^{\# 1,2}$, SHADAN ALI ${ }^{\# 3}$, SANJEEV BANERJEE ${ }^{\# 1}$, BIN BAO ${ }^{1}$, YIWEI LI ${ }^{1}$, ASFAR $^{2}$ \\ S. AZMI ${ }^{1}$, MURRAY KORC ${ }^{4}$, and FAZLUL H. SARKAR ${ }^{1,3,{ }^{*}}$ \\ ${ }^{1}$ Department of Pathology, Karmanos Cancer Institute, Wayne State University School of \\ Medicine, Detroit, Michigan \\ ${ }^{2}$ Department of Biochemistry and Molecular Biology, Bengbu Medical College, Anhui, P.R. China \\ ${ }^{3}$ Department of Oncology, Karmanos Cancer Institute, Wayne State University School of \\ Medicine, Detroit, Michigan \\ ${ }^{4}$ Department of Medicine, Indiana University, Indianapolis, Indiana \\ \# These authors contributed equally to this work.
}

\begin{abstract}
Pancreatic ductal adenocarcinoma (PDAC) is one of the most frequently diagnosed cancers and the fourth leading cause of cancer-related death in the United States, suggesting that there is an urgent need to design novel strategies for achieving better treatment outcome of patients diagnosed with PDAC. Our previous study has shown that activation of Notch and NF-KB play a critical role in the development of PDAC in the compound K-Ras ${ }^{\mathrm{G} 12 \mathrm{D}}$ and Ink4a/Arf deficient transgenic mice. However, the exact molecular mechanism by which mutated K-Ras and Ink4a/ Arf deficiency contribute to progression of PDAC remains largely elusive. In the present study, we used multiple methods, such as real-time RT-PCR, Western blotting assay, and immunohistochemistry to gain further mechanistic insight. We found that the deletion of Ink4a/ Arf in K-Ras ${ }^{G 12 D}$ expressing mice led to high expression of PDGF-D signaling pathway in the tumor and tumor-derived cell line (RInk-1 cells). Furthermore, PDGF-D knock-down in RInk-1 cells resulted in the inhibition of pancreatosphere formation and down-regulation of EZH2, CD44, EpCAM, and vimentin. Moreover, we demonstrated that epithelial-mesenchymal transition (EMT) was induced in the compound mice, which is linked with aggressiveness of PDAC. In addition, we demonstrated that tumors from compound transgenic mice have higher expression of cancer stem cell (CSC) markers. These results suggest that the acquisition of EMT phenotype and induction of CSC characteristics could be linked with the aggressiveness of PDAC mediated in part through the activation of PDGF-D, signaling.
\end{abstract}

\begin{abstract}
Pancreatic ductal adenocarcinoma (PDAC) is one of the most frequent malignancies, which is the fourth leading cause of cancer-related death with an estimated 43,920 new cases and 37,390 deaths expected in the United States in 2012 (Siegel et al., 2012). Although pancreatic cancer is currently treated with surgery, radiation therapy, and chemotherapy, the high mortality rate suggests lack of effective therapies. Therefore, the management of
\end{abstract}

(C) 2012 Wiley Periodicals, Inc.

"Correspondence to: Department of Pathology, Karmanos Cancer Institute, Wayne State University School of Medicine, 740 HWCRC, 4100 JohnR Street, Detroit, MI 48201. fsarkar@med.wayne.edu.

Conflicts of interest: The authors have no conflicts of interest of a financial nature or otherwise. 
PDAC becomes difficult because the disease is diagnosed late in its development, which is in part due to the absence of early specific symptoms and the lack of early detection modality (Siegel et al., 2012). This disappointing outcome strongly suggest that elucidating the molecular mechanism(s) by which pancreatic cancer arises and progresses is necessary for the development of newer therapies to improve the treatment outcome of patients diagnosed with PDAC.

A number of studies have shown that K-Ras mutations are observed in more than $90 \%$ of pancreatic cancer, indicating its potential role in the development of PDAC (Deramaudt and Rustgi, 2005). It has been reported that the most common K-Ras mutation is on codon 12 (K-Ras ${ }^{G 12 D}$ ) in PDAC (Deramaudt and Rustgi, 2005). Thus, to recapitulate the progression of PDAC, a number of mouse models of PDAC have been generated by targeting a conditionally mutated K-Ras ${ }^{\mathrm{G} 12 \mathrm{D}}$ in the pancreas (Johnson et al., 2001; Brembeck et al., 2003; Gidekel Friedlander et al., 2009; Ji et al., 2009; Hanlon et al., 2010). For example, one compound mouse model containing activated K-Ras and Ink4a/Arf deficiency (KCI) has been shown to cooperate in producing metastatic PDAC (Aguirre et al., 2003). Our previous study has shown that activation of Notch and NF-KB were involved in the development of PDAC in the compound K-Ras ${ }^{\mathrm{G} 12 \mathrm{D}}$ and Ink4a/Arf deficient transgenic mice (Wang et al., 2011a). Recently, we found over-expression of several miRNAs, such as miR-21, miR-221, miR-27, and miR-155, and down-regulation in the expression of multiple miRNAs including miR-216a, miR-216b, miR-217, and miR-146a in tumors derived from the compound KRas $^{\mathrm{G} 12 \mathrm{D}}$ and Ink4a/Arf deficient transgenic mice (Ali et al., 2011). However, the underlying molecular mechanisms by which activated K-Ras and Ink4a/Arf deficiency causes PDAC and further aggressiveness are largely unknown.

Emerging evidence suggest that epithelial-to-mesenchymal transition (EMT) plays a key role in tumor aggressiveness by increasing cancer cell metastasis (Thiery et al., 2009). The EMT is a process, whereby epithelial cells acquire mesenchymal cell characteristic. For example, during EMT, epithelial cell phenotype markers such as E-cadherin is downregulated, and mesenchymal markers including Vimentin, Slug, Snail, ZEB1, and ZEB2 are up-regulated (Thiery et al., 2009). These changes leads to increased cell migration and invasion capacity consistent with tumor aggressiveness (Thiery et al., 2009). Recently, it has been demonstrated that the acquisition of EMT is reminiscent of cancer stem cells (CSC) which contributes to the progression of human cancers (Polyak and Weinberg, 2009; Kong et al., 2011).

Accumulated evidence has demonstrated that platelet derived growth factor-D (PDGF-D) plays critical roles in the development and progression of human cancers through regulating many cellular processes, including invasion and angiogenesis mediated by activating its cognate receptor platelet-derived growth factor receptor- $\beta$ (PDGFR- $\beta$ ) (Wang et al., 2010). Our previous studies have shown that PDGF-D contributes to EMT in prostate cancer (Kong et al., 2008b, 2009). We also reported that PDGF-D promotes cell growth and angiogenesis through activation of Notch and NF-KB signaling in breast cancer and pancreatic cancer (Wang et al., 2007). However, the question remains whether PDGF-D is involved in the development of PDAC in the compound transgenic mice with activated K-Ras and Ink4a/ Arf deficiency or not and whether it is due to the acquisition of EMT phenotype. Emerging evidence suggest that the over-expression of PDGF-D might cause normal cells to dedifferentiate to the stem cell-like state which could be reversed by siRNA approach in KCI-derived RInk-1 cells, suggesting that additional mechanistic understanding of PDGF-D regulation and finding novel agents that could inactivate PDGF-D signaling would become a novel strategy for the treatment of PDAC. 
In the present study, we investigated whether EMT and CSC phenotypes are present or not in the mouse tumors (and tumor-derived RInk-1 cells) developed in the compound transgenic mice with activated K-Ras and Ink4a/Arf deficiency. Consistent with our previous findings, the deletion of Ink4a/Arf in K-Ras expressing mice led to the development of PDAC, and here we show, for the first, that tumor development in this model is in part mediated through the acquisition of EMT and CSC phenotype.

\section{Materials and Methods}

\section{Ethics statement}

This study was conducted in accordance with the recommendations in the guidelines for the care and use of laboratory animals of the National Institutes of Health. All animal studies and procedures were approved by the Wayne State University Animal Care and Use Committee. All studies involving mice were performed under Animal Investigation Committee (AIC)-approved protocols.

\section{Mouse strains}

The LSL-K-Ras ${ }^{\text {G12D }}$ strain was bred to the following strains: Pdx1-Cre, INK4a/Arf lox/lox as previously described (Wang et al., 2011a). Pancreata were collected and processed for analysis. Tumor tissues harvested from this experiment was used for histologic, immunohistochemical, real-time RT-PCR, and Western blotting analysis.

\section{Genotyping}

For genotyping, genomic DNA was extracted from tail cuttings using the REDExtract-NAmp Tissue PCR kit (Sigma-Aldrich, St. Louis, MO). Three PCR reactions were carried out for each animal to investigate the presence of the correct oncogenic K-Ras, p16, and Pdx1Cre transgene constructs, respectively.

\section{Cells culture}

Mouse pancreatic cancer cell line RInk-1 was chosen for this study. RInk-1 murine pancreatic tumor cell line was generated from the LSL-K-Ras ${ }^{\mathrm{G} 12 \mathrm{D}}$;Pdx1-Cre;INK4a/Arf (KCI) mice as described previously (Seeley et al., 2009).

\section{Immunohistochemistry}

Histopathologic analysis of pancreata was carried out. The expression of PDGF-D was detected in histologic sections of tumor. Sections were cut from formalin-fixed, paraffinembedded tissue blocks; collected on 3-ethoxy-aminoethyl-silane-treated slides; and allowed to dry overnight at $37^{\circ} \mathrm{C}$. Sections were dewaxed in xylene, rehydrated through graded concentrations of ethanol to distilled water, immersed in $10 \mathrm{mmol} / \mathrm{L}$ citrate buffer ( $\mathrm{pH} 6.0$ ), and processed in a thermostatic water bath for $40 \mathrm{~min}$ at $98^{\circ} \mathrm{C}$ for antigen retrieval. AntiPDGF-D antibody was applied on three slides for each case, and incubations were done overnight at room temperature in a humidified atmosphere followed by a 30-min incubation of secondary antibody. Slides were then incubated with streptavidin peroxidase and visualized using the 3,3'-diaminobenzidine chromogen (Lab Vision Corp., Fremont, CA).

Transfection of PDGF-D siRNA: RInk-1 murine pancreatic tumor cell line was plated in sixwell plate, cultured overnight and transfected with $10 \mu \mathrm{g}$ of PDGF-D siRNA or scramble control siRNA (Santa Cruz Biotechnology, Santa Cruz, CA) by DharmaFECT (Thermo Scientific, Chicago, IL) following the manufacturer's protocol. After $48 \mathrm{~h}$ of transfection, the cells were collected for Western blot analysis and/or RT-PCR for assessing the expression of genes at the mRNA levels. 


\section{Sphere formation assay}

Sphere formation assay was performed to assess the capacity of CSC self-renewal following procedures described by us previously (Bao et al., 2011a). Briefly, single cell suspensions of cells were plated on ultra low adherent wells of six-well plate (Corning, Lowell, MA) at 500 cells/well in sphere formation medium (1:1 DMEM/F12 medium supplemented with B-27 and N-2; Invitrogen, Carlsbad, CA) for 10 days. Moreover, PDGF-D siRNA was added to the wells, and compared with control siRNA treated wells. After 7 days, the spheres termed as "pancreatospheres" were collected by centrifugation ( $300 \mathrm{~g}, 5 \mathrm{~min})$, and counted under a microscope.

\section{Real-time reverse transcription-PCR analysis for gene expression studies}

The total RNA from animal tissues was isolated by Trizol (Invitrogen, Carlsbad, CA) and purified by RNeasy Mini Kit and RNase-free DNase Set (QIAGEN, Valencia, CA) according to the manufacturer's protocols. One microgram of total RNA from each sample was subjected to first strand cDNA synthesis using High Capacity RNA-to-cDNA kit (Applied Biosystems, Foster City, CA) in a total volume of $20 \mu \mathrm{l}$, including reverse transcriptase enzyme mix. RT reaction was performed at $37^{\circ} \mathrm{C}$ for $60 \mathrm{~min}$, followed by $95^{\circ} \mathrm{C}$ for $5 \mathrm{~min}$. The primers used in the PCR reaction were Lin28B, CD44, EZH2, PDGF-D, and PDGFR $\beta$, were synthesized using reagents obtained from Invitrogen (Grand Island, NY). Real-time PCR amplifications were performed as described previously by our laboratory (Wang et al., 2006).

\section{Western blot analysis}

RInk-1 cells and the animal tissues were homogenized and sonicated in $62 \mathrm{mM}$ Tris- $\mathrm{HCl}$ and 2\% SDS. The protein concentration was determined using the Bio-Rad assay system (Bio-Rad, Hercules, CA). Total proteins were fractionated using SDS-PAGE and transferred onto nitrocellulose membrane for Western blotting as described by our laboratory earlier (Wang et al., 2006). Antibodies against CD44, EpCAM, Lin28B, and EZH2 were purchased from Cell Signaling Technology (Beverly, MA). Antibodies against ZEB2, PDGFR $\beta$, RPS6, and E-cadherin were purchased from Santa Cruz Biotechnology. Antibody against PDGF-D was purchased from Invitrogen. Antibody against $\beta$-actin was acquired from Sigma Chemicals (St. Louis, MO). All secondary antibodies were obtained from Pierce (Rockford, IL).

\section{Statistical analysis}

The statistical significance of differential findings between experimental groups and control groups was statistically evaluated using GraphPad StatMate software (GraphPad Software, Inc., San Diego, CA). $P$-values lower than 0.05 were considered statistically significant.

\section{Results \\ PDGF-D signaling pathway is highly expressed in Pdx1-Cre;LSL-K-Ras ${ }^{\mathrm{G} 12 \mathrm{D} \text {;Ink4a/Arf }}$ mouse}

We have previously shown that PDGF-D plays a critical role in the development and progression of pancreatic cancer (Wang et al., 2007). To determine whether PDGF-D is involved in the progression of PDAC in the mutated K-Ras mice, we examined the expression of PDGF-D pathway in a murine model. In this model, oncogenic K-Ras (K$\operatorname{Ras}^{\mathrm{G} 12 \mathrm{D}}$ ) is knocked-in into its own locus and transcriptionally silenced due to the insertion of a LoxP-Stop-LoxP element (LSL). When LSL-K-Ras ${ }^{\text {G12D }}$ mice are bred with transgenic mice which express Cre-recombinase under the control of the Pdx1 promoter, expression of Cre-recombinase in pancreatic progenitor cells removes the floxed transcriptional STOP 
cassette, leading to the activation of the oncogenic K-Ras allele downstream of its own endogenous promoter. Our previously published study has shown that pancreatic tumors did not develop in 24- and 30-week-old Pdx1-re:deletion of Ink4a/Arf (CI) and LSL- $\underline{\mathrm{K}}$ $\underline{\operatorname{Ras}}^{\mathrm{G} 12 \mathrm{D}}:$ Pdx1-Cre (KC) mice, respectively (Wang et al., 2011a). However, mice from LSL$\underline{\text { K-Ras }}{ }^{G 12 D}$ :Pdx1-Cre: Ink4a/Arf (KCI) animals were found to form solid pancreatic tumors ranging in diameter from 4 to $10 \mathrm{~mm}$ between 45 and 80 days (Wang et al., 2011a).

It has been reported that PDGF-D signaling pathway plays pivotal roles in many human cancers including pancreatic cancer (Wang et al., 2009, 2011a). Therefore, we examined the levels of PDGF-D expression in our transgenic mice tissues. We found that PDGF-D signaling was activated in KCI mice compared with $\mathrm{KC}$ and $\mathrm{CI}$ mice (Fig. 1). The expression of PDGF-D was up-regulated both at the mRNA and protein levels in KCI mice (Fig. 1A,B). Moreover, we found that PDGFR $\beta$ was up-regulated in KCI mice (Fig. 1B). To further confirm these results, we assessed the expression of PDGF-D by IHC in animal tissues. Consistent with the Western blotting analysis, we found that the expression of PDGF-D was increased in KCI mice compared to tissues from KC and CI mice (Fig. 2).

\section{PDGF-D siRNA inhibited EMT, CSCs, and pancreatospheres formation in PC cells}

In order to investigate the role of PDGF-D in the regulation of EMT, CSCs markers, and CSC self-renewal capacity, we conducted the PDGF-D siRNA transfection experiments using RInk-1 cells. We found that knock-down of PDGF-D by siRNA transfection led to a significantly decrease in the expression of PDGF-D but most interestingly, the knock-down of PDGF-D led to decreased expression of vimentin, EZH2, EpCAM, CD44 expression as documented by Western blot analysis (Fig. 3A). Similarly, we also found reduced mRNA expression of EZH2, CD44, and Lin28B by RT-PCR (Fig. 3B). In addition, we found partial elimination of pancreatospheres formation capacity of RInk-1 cells (Fig. 3C), suggesting that PDGF-D knock-down could be useful for targeted elimination of EMT phenotypic cells or the killing of CSCs.

\section{Expression of stem cell markers in the tissues from $\mathrm{KCl}$ mouse}

Our previous studies have shown that EMT is mechanistically linked with stem cell signatures in human cancers including PDAC (Bao et al., 2011b,c; Kong et al., 2008b, 2009). To further determine whether tissues with EMT phenotype from KCI mice have stem cell characteristics, we assessed the expression of stem cell markers in the tissues form $\mathrm{KC}$, CI, and KCI mice by real-time RT-PCR and Western blot analysis. The results from realtime RT-PCR showed 40-fold to 500-fold increase in the mRNA levels of these stem cell markers (Fig. 4A). Western blotting analysis also demonstrated that the protein expression of Lin28B, EZH2, and EpCAM were significantly higher in KCI mice compared to KC and CI mice (Fig. 4B). These results suggest the existence of stem cell characteristics in the tumors from KCI mice.

\section{Induction of EMT in the tissues from $\mathrm{KCl}$ mouse}

Our previous studies have demonstrated that PDGF-D could induce EMT in a variety of human cancers including prostate cancer and breast cancer (Kong et al., 2008b; Sethi et al., 2011). It is well known that EMT is involved in tumor migration, invasion, and metastasis. Moreover, previous report has shown that KCI mice have metastasis to multiple organ sites (Aguirre et al., 2003), suggesting that the tumors developed in the KCI mice is highly aggressive. Therefore, it is logical to hypothesize that the expression of PDGF-D could lead to the acquisition of EMT phenotype in KCI mice, resulting in tumor metastasis. To test this hypothesis, we examined EMT markers to investigate whether KCI mice tumors underwent EMT due to over-expression of PDGF-D. We found that E-cadherin expression was significantly reduced, whereas the expression of Vimentin and ZEB2 was significantly 
increased (Fig. 4C), suggesting that the expression of these factors may be important for EMT induction in KCI mice. The results from tumor specimens were also consistent with data from tumor-derived RInk-1 cells.

\section{Discussion}

PDAC is an aggressive malignancy and currently it is the fourth leading cause of cancerrelated deaths in the United States (Siegel et al., 2012). Although novel treatment strategies such as chemotherapy, targeted therapy, and radiation therapy is the standard of care, the median survival period is 4-6 months and the 5-year survival rate is $<5 \%$ (Siegel et al., 2012). Since patients diagnosed with PDAC have poor prognosis and high mortality, there is an urgent need to investigate the exact molecular mechanism by which PDAC arises and rapidly metastasize to the lymphatic system and distant organs (Siegel et al., 2012). Accumulated evidence has demonstrated that transgenic mice are ideal models to identify key signaling pathways associated with PDAC (Grippo and Tuveson, 2010).

The growing body of literature has demonstrated that PDGF-D may function as a pivotal player in human tumorigenesis through regulation of key cellular processes including cell proliferation, apoptosis, migration, invasion, angiogenesis, and metastasis (Ustach and Kim, 2005; Kong et al., 2008a; Ustach et al., 2010; Wang et al., 2010; Ahmad et al., 2011). Upregulated expression of PDGF-D has been observed in human malignancies such as lung, renal, ovarian, and brain cancer, suggesting the potential oncogenic activity of PDGF-D in human cancers (Ustach and Kim, 2005; Kong et al., 2008b; Wang et al., 2009; Ahmad et al., 2011). We recently found that PDGF-D is highly expressed in human pancreatic adenocarcinoma specimens, in chronic pancreatitis associated with pancreatic adenocarcinoma, and in different human pancreatic cancer cell lines (Wang et al., 2007). Consistent with these results, in the present study, we used the compound KCI mice, which recapitulated most features of human PDAC to determine whether PDGF-D signaling pathway could be mechanistically linked with the development of PDAC and its aggressiveness. Indeed, we found over-expression of PDGF-D signaling pathway in the tumors of KCI mice as well as in tumor-derived RInk-1 cells.

In recent years, accumulated evidence has suggested that EMT plays a critical role in PDAC associated with increased invasion, metastasis, and chemo-resistance (Cano et al., 2010; Wang et al., 2011b). Moreover, EMT has been demonstrated in human resected PDAC specimens, and tumorigenic cells with mesenchymal features show a positive correlation with high-grade tumors in PDAC (Rasheed et al., 2010). The acquisition of EMT phenotype is also linked with prognosis in PDAC (Iyer et al., 2007). Specifically, PDAC with more mesenchymal features has worse survival and an increased numbers of metastases (Iyer et al., 2007). In PDAC, the expression of Snail and ZEB1 is also associated with tumor grade and predicts poor prognosis (Hotz et al., 2007). Several distinct signaling pathways have been identified in cells with EMT phenotype, including WNT, Notch, Hedgehog, and transforming growth factor-beta (TGF- $\beta$; Cano et al., 2010). Recently, we have demonstrated that PDGF-D signaling pathway is also involved in the acquisition of EMT (Kong et al., 2008b). Our previous studies have also shown that PDGF-D induced acquisition of the EMT phenotype occurred through repression of miR-200 in prostate cancer (Kong et al., 2009). More recently, EMT in breast cell lines was found to be mediated through PDGF-D pathway (Devarajan et al., 2011). Here, for the first time, we showed the existence of EMT in KCI mice with high expression of PDGF-D, suggesting that PDGF-D might be involved in the acquisition of EMT features in the development and progression of PDAC in the KCI mice. 
Our recent studies have shown that the processes of EMT are linked with CSC characteristics in pancreatic cancer and prostate cancer, which is consistent with recent reports from other groups (Mani et al., 2008; Kong et al., 2008b; Scheel et al., 2011; Bao et al., 2011b,c). The existence of CSCs provides an explanation for the clinical observation that tumor cannot be cured because of subsequent tumor recurrence and metastasis due to the survival of CSCs. Therefore, the killing or inactivation of CSCs might provide a new specific approach for eliminating cells that are the root cause of drug resistance, tumor recurrence, and metastasis. Our previous studies have shown that over-expression of PDGFD leads to the acquisition of EMT phenotype, which was also consistent with stem cell characteristics such as self-renewal capacity, and expression of stem cell markers in prostate cancer (Kong et al., 2008b, 2009). More recently, it was found that tissue-resident stem cells interact with the cancer microenvironment via PDGF-D, induce EMT in the breast cancer cells in a paracrine fashion, thereby increasing the number of CSCs leading to increased tumor growth in a PDGF dependent manner (Devarajan et al., 2011). In the current study, we show that the tumors from KCI mice over-expressed PDGF-D which is linked with the acquisition of EMT and CSC phenotype, suggesting that PDGF-D could play a critical role in promoting tumor metastasis and aggressiveness.

Therefore, targeting PDGF-D signaling pathway or EMT-targeted (CSC targets) could be a novel strategy for the treatment of PDAC. In our current study, we have demonstrated that the decreased PDGF-D expression using PDGF-D siRNA transfection in RInk-1 cell line led to decreased protein expressions of CSC markers such as EZH2, CD44, and EpCAM. Moreover, EZH2 deficiency led to increased expression of EMT markers such as vimentin, and ZEB2, with simultaneous reduction in E-cadherin expression, suggesting that these CSCs/EMT markers are involved in the regulation of PDGF-D mediated tumor cell aggressiveness, and that inactivation of PDGF-D could contribute to inhibit tumor aggressiveness through deregulation of EMT and CSC markers and also the formation of pancreatospheres.

In summary, our results suggest that the activation of PDGF-D, together with the acquisition of EMT and CSC features is mechanistically linked with the development and progression of PDAC in the compound $\mathrm{K}-\mathrm{Ras}{ }^{\mathrm{G} 12 \mathrm{D}}$ and Ink4a/Arf deficient transgenic mice. Recently, we reported that PDGF-D could regulate the Notch signaling pathway, which induces EMT in pancreatic cancer (Wang et al., 2007; Bao et al., 2011c). Given the importance of PDGF$\mathrm{D}$ in tumor cell growth, migration, invasion, angiogenesis, and metastasis and its cross-talks with many signaling pathways in human malignancies, we propose a hypothetical pathway by which the deletion of Ink4a/Arf in K-Ras ${ }^{G 12 D}$ expressing mice caused high expression of PDGF-D, leading to the up-regulation of Notch pathway, acquisition of EMT and development of PDAC (Fig. 5). Overall, our study suggests that PDGF-D or EMT-targeting (CSC-targeting) agents could become a therapeutic strategy for the treatment of PDAC with better survival outcome, and thus further investigation is warranted.

\section{Acknowledgments}

The authors' work was funded by grants from the National Cancer Institute, NIH R01CA131151, R01CA132794, and R01CA154321 to F.H.S. This work was also supported by grants from the NSFC (No. 81172087). We also sincerely thank both Puschelberg and Guido foundation for their generous contributions to our research.

Contract grant sponsor: National Cancer Institute;

Contract grant numbers: R01CA131151, R01CA132794, 1R01CA154321.

Contract grant sponsor: NIH.

Contract grant sponsor: NSFC; 
Contract grant number: 81172087 .

\section{Literature Cited}

Aguirre AJ, Bardeesy N, Sinha M, Lopez L, Tuveson DA, Horner J, Redston MS, DePinho RA. Activated Kras and Ink4a/Arf deficiency cooperate to produce metastatic pancreatic ductal adenocarcinoma. Genes Dev. 2003; 17:3112-3126. [PubMed: 14681207]

Ahmad A, Wang Z, Kong D, Ali R, Ali S, Banerjee S, Sarkar FH. Platelet-derived growth factor-D contributes to aggressiveness of breast cancer cells by up-regulating Notch and NF-kappaB signaling pathways. Breast Cancer Res Treat. 2011; 126:15-25. [PubMed: 20379844]

Ali S, Banerjee S, Logna F, Bao B, Philip PA, Korc M, Sarkar FH. Inactivation of Ink4a/Arf leads to deregulated expression of miRNAs in K-Ras transgenic mouse model of pancreatic cancer. J Cell Physiol. 2011; 227:3373-3380. [PubMed: 22213426]

Bao B, Ali S, Kong D, Sarkar SH, Wang Z, Banerjee S, Aboukameel A, Padhye S, Philip PA, Sarkar FH. Anti-tumor activity of a novel compound-CDF is mediated by regulating miR-21, miR-200, and PTEN in pancreatic cancer. PLoS One. 2011a; 6:e17850. [PubMed: 21408027]

Bao B, Wang Z, Ali S, Kong D, Banerjee S, Ahmad A, Li Y, Azmi AS, Miele L, Sarkar FH. Overexpression of FoxM1 leads to epithelial-mesenchymal transition and cancer stem cell phenotype in pancreatic cancer cells. J Cell Biochem. 2011b; 112:2296-2306. [PubMed: 21503965]

Bao B, Wang Z, Ali S, Kong D, Li Y, Ahmad A, Banerjee S, Azmi AS, Miele L, Sarkar FH. Notch-1 induces epithelial-mesenchymal transition consistent with cancer stem cell phenotype in pancreatic cancer cells. Cancer Lett. 2011c; 63:26-36. [PubMed: 21463919]

Brembeck FH, Schreiber FS, Deramaudt TB, Craig L, Rhoades B, Swain G, Grippo P, Stoffers DA, Silberg DG, Rustgi AK. The mutant K-ras oncogene causes pancreatic periductal lymphocytic infiltration and gastric mucous neck cell hyperplasia in transgenic mice. Cancer Res. 2003; 63:2005-2009. [PubMed: 12727809]

Cano CE, Motoo Y, Iovanna JL. Epithelial-to-mesenchymal transition in pancreatic adenocarcinoma. Scientific World J. 2010; 10:1947-1957.

Deramaudt T, Rustgi AK. Mutant KRAS in the initiation of pancreatic cancer. Biochim Biophys Acta. 2005; 1756:97-101. [PubMed: 16169155]

Devarajan E, Song YH, Krishnappa S, Alt E. Epithelial-mesenchymal transition in breast cancer lines is mediated through PDGF-D released by tissue-resident stem cells. Int J Cancer. 2011; 131:10231031. [PubMed: 22038895]

Gidekel Friedlander SY, Chu GC, Snyder EL, Girnius N, Dibelius G, Crowley D, Vasile E, DePinho RA, Jacks T. Context-dependent transformation of adult pancreatic cells by oncogenic K-Ras. Cancer Cell. 2009; 16:379-389. [PubMed: 19878870]

Grippo PJ, Tuveson DA. Deploying mouse models of pancreatic cancer for chemoprevention studies. Cancer Prev Res (Phila). 2010; 3:1382-1387. [PubMed: 21045161]

Hanlon L, Avila JL, Demarest RM, Troutman S, Allen M, Ratti F, Rustgi AK, Stanger BZ, Radtke F, Adsay V, Long F, Capobianco AJ, Kissil JL. Notch1 functions as a tumor suppressor in a model of K-ras-induced pancreatic ductal adenocarcinoma. Cancer Res. 2010; 70:4280-4286. [PubMed: 20484026]

Hotz B, Arndt M, Dullat S, Bhargava S, Buhr HJ, Hotz HG. Epithelial to mesenchymal transition: Expression of the regulators snail, slug, and twist in pancreatic cancer. Clin Cancer Res. 2007; 13:4769-4776. [PubMed: 17699854]

Iyer RV, Gibbs J, Kuvshinoff B, Fakih M, Kepner J, Soehnlein N, Lawrence D, Javle MM. A phase II study of gemcitabine and capecitabine in advanced cholangiocarcinoma and carcinoma of the gallbladder: A single-institution prospective study. Ann Surg Oncol. 2007; 14:3202-3209. [PubMed: 17705089]

Ji B, Tsou L, Wang H, Gaiser S, Chang DZ, Daniluk J, Bi Y, Grote T, Longnecker DS, Logsdon CD. Ras activity levels control the development of pancreatic diseases. Gastroenterology. 2009; 137:1072-1082. [PubMed: 19501586] 
Johnson L, Mercer K, Greenbaum D, Bronson RT, Crowley D, Tuveson DA, Jacks T. Somatic activation of the K-ras oncogene causes early onset lung cancer in mice. Nature. 2001; 410:11111116. [PubMed: 11323676]

Kong D, Banerjee S, Huang W, Li Y, Wang Z, Kim HR, Sarkar FH. Mammalian target of rapamycin repression by 3,3'-diindolylmethane inhibits invasion and angiogenesis in platelet-derived growth factor-D-overexpressing PC3 cells. Cancer Res. 2008a; 68:1927-1934. [PubMed: 18339874]

Kong D, Wang Z, Sarkar SH, Li Y, Banerjee S, Saliganan A, Kim HR, Cher ML, Sarkar FH. Plateletderived growth factor-D overexpression contributes to epithelial-mesenchymal transition of PC3 prostate cancer cells. Stem Cells. 2008b; 26:1425-1435. [PubMed: 18403754]

Kong D, Li Y, Wang Z, Banerjee S, Ahmad A, Kim HR, Sarkar FH. miR-200 regulates PDGF-Dmediated epithelial-mesenchymal transition, adhesion, and invasion of prostate cancer cells. Stem Cells. 2009; 27:1712-1721. [PubMed: 19544444]

Kong D, Li Y, Wang Z, Sarkar FH. Cancer stem cells and epithelial-to-mesenchymal transition (EMT)-phenotypic cells: Are they cousins or twins? Cancers (Basel). 2011; 3:716-729. [PubMed: 21643534]

Mani SA, Guo W, Liao MJ, Eaton EN, Ayyanan A, Zhou AY, Brooks M, Reinhard F, Zhang CC, Shipitsin M, Campbell LL, Polyak K, Brisken C, Yang J, Weinberg RA. The epithelialmesenchymal transition generates cells with properties of stem cells. Cell. 2008; 133:704-715. [PubMed: 18485877]

Polyak K, Weinberg RA. Transitions between epithelial and mesenchymal states: Acquisition of malignant and stem cell traits. Nat Rev Cancer. 2009; 9:265-273. [PubMed: 19262571]

Rasheed ZA, Yang J, Wang Q, Kowalski J, Freed I, Murter C, Hong SM, Koorstra JB, Rajeshkumar NV, He X, Goggins M, Iacobuzio-Donahue C, Berman DM, Laheru D, Jimeno A, Hidalgo M, Maitra A, Matsui W. Prognostic significance of tumorigenic cells with mesenchymal features in pancreatic adenocarcinoma. J Natl Cancer Inst. 2010; 102:340-351. [PubMed: 20164446]

Scheel C, Eaton EN, Li SH, Chaffer CL, Reinhardt F, Kah KJ, Bell G, Guo W, Rubin J, Richardson AL, Weinberg RA. Paracrine and autocrine signals induce and maintain mesenchymal and stem cell states in the breast. Cell. 2011; 145:926-940. [PubMed: 21663795]

Seeley ES, Carriere C, Goetze T, Longnecker DS, Korc M. Pancreatic cancer and precursor pancreatic intraepithelial neoplasia lesions are devoid of primary cilia. Cancer Res. 2009; 69:422-430. [PubMed: 19147554]

Sethi S, Sarkar FH, Ahmed Q, Bandyopadhyay S, Nahleh ZA, Semaan A, Sakr W, Munkarah A, AliFehmi R. Molecular markers of epithelial-to-mesenchymal transition are associated with tumor aggressiveness in breast carcinoma. Transl Oncol. 2011; 4:222-226. [PubMed: 21804917]

Siegel R, Naishadham D, Jemal A. Cancer statistics, 2012. CA Cancer J Clin. 2012; 62:10-29. [PubMed: 22237781]

Thiery JP, Acloque H, Huang RY, Nieto MA. Epithelial-mesenchymal transitions in development and disease. Cell. 2009; 139:871-890. [PubMed: 19945376]

Ustach CV, Kim HR. Platelet-derived growth factor D is activated by urokinase plasminogen activator in prostate carcinoma cells. Mol Cell Biol. 2005; 25:6279-6288. [PubMed: 15988036]

Ustach CV, Huang W, Conley-LaComb MK, Lin CY, Che M, Abrams J, Kim HR. A novel signaling axis of matriptase/PDGF-D/ss-PDGFR in human prostate cancer. Cancer Res. 2010; 70:96319640. [PubMed: 21098708]

Wang Z, Banerjee S, Li Y, Rahman KM, Zhang Y, Sarkar FH. Down-regulation of notch-1 inhibits invasion by inactivation of nuclear factor-kappaB, vascular endothelial growth factor, and matrix metalloproteinase-9 in pancreatic cancer cells. Cancer Res. 2006; 66:2778-2784. [PubMed: 16510599]

Wang Z, Kong D, Banerjee S, Li Y, Adsay NV, Abbruzzese J, Sarkar FH. Down-regulation of platelet-derived growth factor-D inhibits cell growth and angiogenesis through inactivation of Notch-1 and nuclear factor-kappaB signaling. Cancer Res. 2007; 67:11377-11385. [PubMed: 18056465]

Wang Z, Kong D, Li Y, Sarkar FH. PDGF-D signaling: A novel target in cancer therapy. Curr Drug Targets. 2009; 10:38-41. [PubMed: 19149534] 
Wang Z, Ahmad A, Li Y, Kong D, Azmi AS, Banerjee S, Sarkar FH. Emerging roles of PDGF-D signaling pathway in tumor development and progression. Biochim Biophys Acta. 2010; 1806:122-130. [PubMed: 20434526]

Wang Z, Banerjee S, Ahmad A, Li Y, Azmi AS, Gunn JR, Kong D, Bao B, Ali S, Gao J, Mohammad RM, Miele L, Korc M, Sarkar FH. Activated K-ras and INK4a/Arf deficiency cooperate during the development of pancreatic cancer by activation of Notch and NF-kappaB signaling pathways. PLoS ONE. 2011a; 6:e20537. [PubMed: 21673986]

Wang Z, Li Y, Ahmad A, Banerjee S, Azmi AS, Kong D, Sarkar FH. Pancreatic cancer: Understanding and overcoming chemoresistance. Nat Rev Gastroenterol Hepatol. 2011b; 8:27-33. [PubMed: 21102532] 

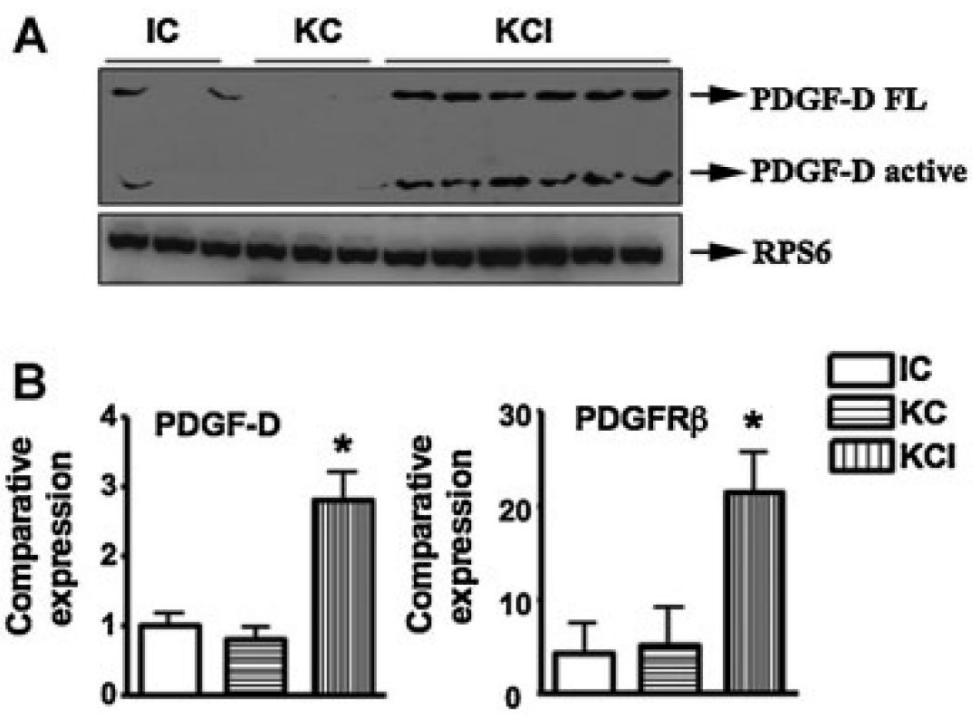

Fig. 1.

PDGF-D pathway is highly activated in KCI mice. A: The expression of PDGF-D was measured at protein levels in KCI mice by Western blotting analysis. B: PDGF-D and PDGFR- $\beta$ mRNA expression was detected in KCI mice by real-time RT-PCR technique. 
IC
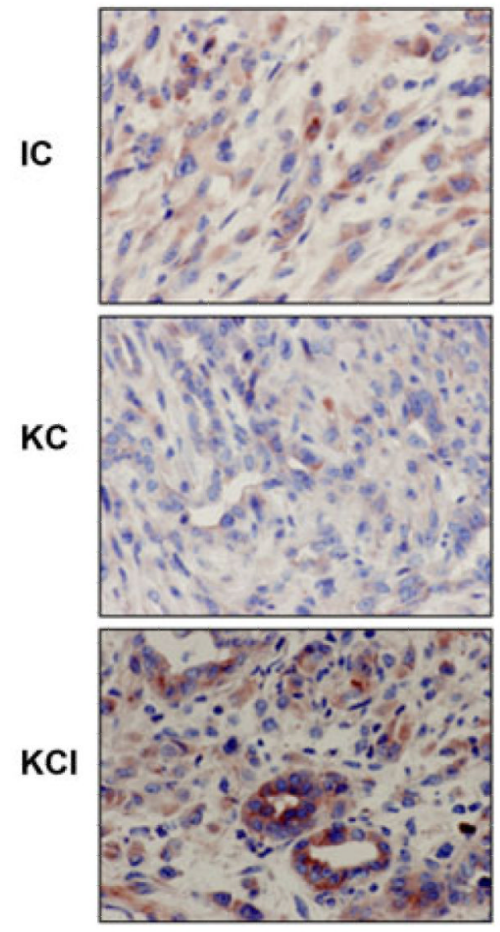

PDGF-D

Fig. 2.

PDGF-D expression is higher in KCI mice. The PDGF-D expression was detected by IHC in animal tissues. We found that expression of PDGF-D was increased in KCI mice compared with tissues from $\mathrm{KC}$ and $\mathrm{CI}$ mice. 


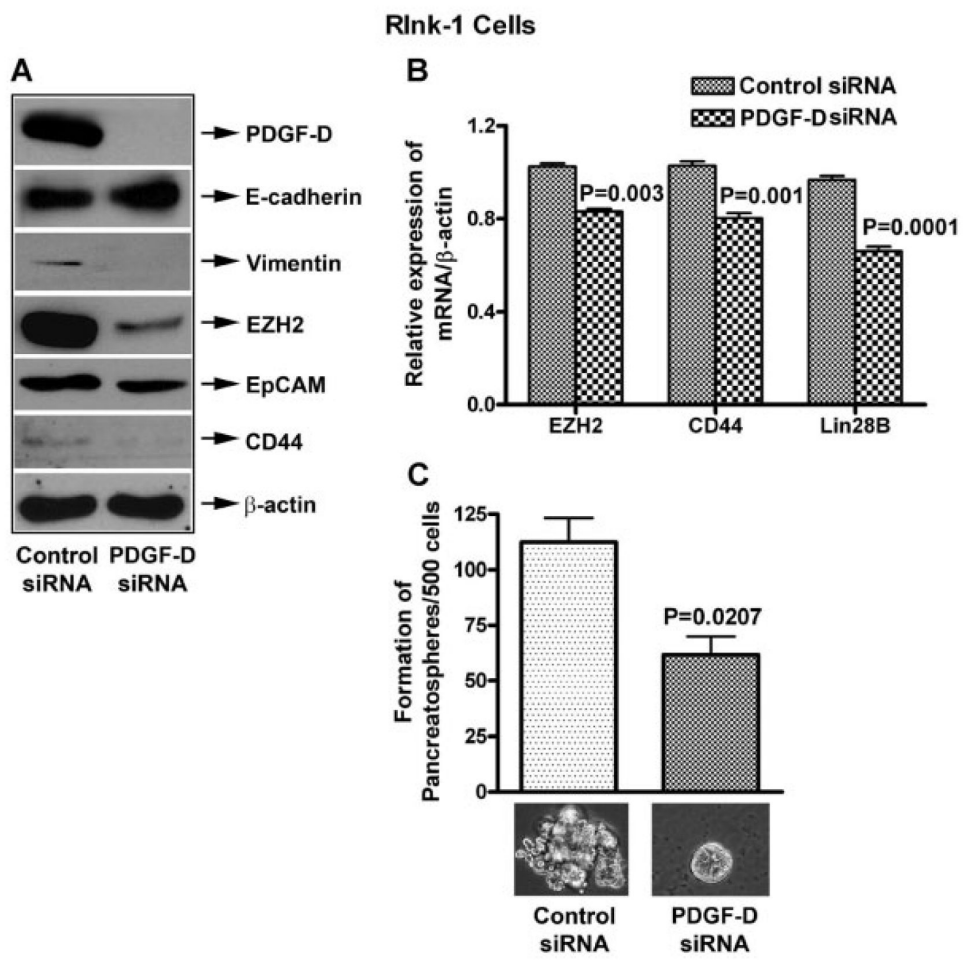

Fig. 3.

PDGF-D siRNA transfection reduces EMT, CSCs markers, and pancreatosphere formation in RInk-1 murine cells. A: PDGF-D siRNA decreased the relative protein levels of Vimentin, EZH2, EpCAM, CD44, and up-regulated E-cadherin. B: It also reduced mRNA levels of EZH2, CD44, and Lin28B, and C: reduced pancreatosphere formation, in RInk-1 cells. 
A

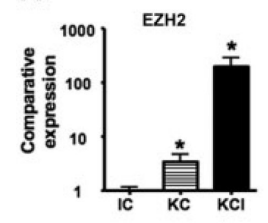

B

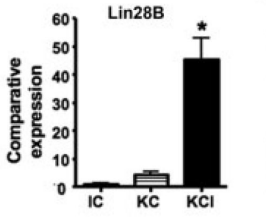

IC $\quad \mathrm{KC} \quad \mathrm{KCI}$

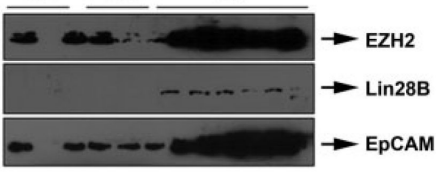

C

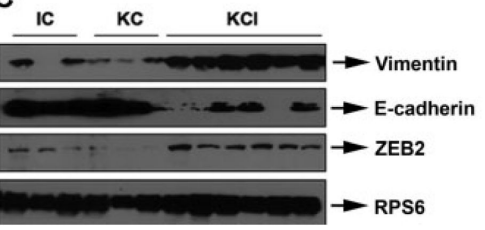

Fig. 4.

The existence of stem cell characteristics, and acquisition of EMT in KCI mice. The expression of stem cell markers in the tissues from KC, CI, and KCI mice was detected by real-time RT-PCR and Western blot analysis, respectively. The expression of EMT markers was measured by Western blotting analysis. We found that E-cadherin expression was significantly reduced and the expression of Vimentin and ZEB2 was highly elevated. 


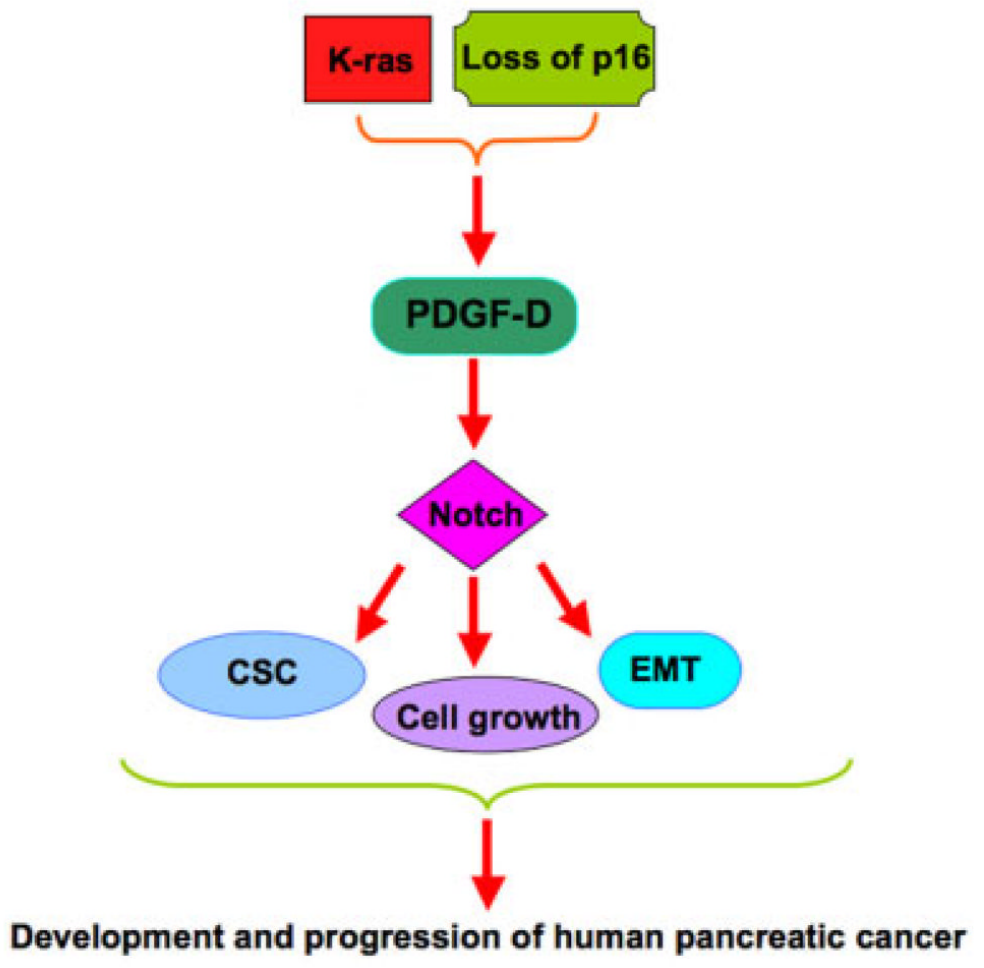

Fig. 5.

The schematic representation of our proposed mechanism of how PDGF-D promotes the development and progression of PDAC in the KCI mice. 